\title{
Efficacy analysis of Traditional Chinese Medicine Syndrome Differentiation on Pneumoconiosis
}

\section{HU Jun 1 , SONG Ping-ping², SUN Xiao-wei², MA Xue-yu³, CHEN}

\section{Yan-xia²}

\author{
${ }^{1}$ Medical College of Qingdao University, Qingdao, Shandong, 266021,China; \\ ${ }^{2}$ Department of Occupational Disease, Qingdao Central Hospital, Qingdao, Shandong, 266042, China; \\ ${ }^{3}$ Department of Traditional Chinese Medicine, Qingdao Central Hospital, Qingdao, Shandong, 266042,China \\ *Foundation items: Project supposed by science and technology of Qingdao (14-2-3-24-nsh)
}

\begin{abstract}
Objective: To study the clinical effect of TCM syndrome differentiation and treatment on pneumoconiosis. Methods: Randomly selected 160 cases of pneumoconiosis patients admitted to our hospital, stratified randomly divided into the experimental group and the control group; The control group was given conventional oxygen and nursing therapy,the experimental group on the basis of the control group was given TCM syndrome differentiation. Two groups of patients had to finished the SGRQ before and after treatment, And use the method of immunofluorescence to check out the content of procollagen type III(PCIII), collagen type IV(IV-C), hyaluronic acid(HA) and laminin(LN) in serum in the two groups of patients before and after treatment. Two groups of clinical effect will been judged in one month. Results: The two groups in age, the cumulative dust length, the content of four parameters of fibrosis in serum, SGRQ each score and total score had no difference(P>0.05); In the two groups after treatment, there is difference on the content of PCIII in serum, respiratory symptoms, sickness influence and total score, with statistical significance $(\mathrm{P}<0.05)$. The experimental treatment efficiency is higher than the control group treatment efficiency. Conclusion: Traditional Chinese Medicine syndrome differentiation and treatment on pneumoconiosis, can delay the development of patients ' condition, have the effect on easing patients' clinical symptoms, and have no obvious adverse reactions.
\end{abstract}

Keywords: Traditional Chinese Medicine(TCM); Syndrome differentiation; Pneumoconiosis; Curative effect analysis

Chinese Library Classification(CLC): R135.2 Document Code:A Article ID:

This article is published under the terms of the Creative Commons Attribution License 4.0 Author(s) retain the copyright of this article. Publication rights with Alkhaer Publications. Published at: http://www.ijsciences.com/pub/issue/2016-11/

DOI: 10.18483/ijSci.1158; Online ISSN: 2305-3925; Print ISSN: 2410-4477 


\section{Introduction}

Pneumoconiosis is due to the long-term inhalation of productive dust in professional activities, and retention in the lung, mainly diffuse fibrosis of lung tissue caused by systemic diseases ${ }^{[1]}$. The main clinical symptoms of respiratory are cough, sputum, chest pain and dyspnea; In addition there are breathing, hemoptysis and some systemic symptom. Pneumoconiosis once occurred, a progressive development, not only endanger the health of the workers exposed with dust and reduce the labor ability and quality of life, and ultimately cause huge economic loss to the country ${ }^{[2-3]}$. At present, It is lack of effective treatment methods in the treatment of pneumoconiosis, application of western medicine treatment of pneumoconiosis is given priority to with glucocorticoid and immunosuppressants at home and abroad, due to its side effect is remarkable, and its treatment effect is not ideal, its application is limited for certain in clinical.

Pneumoconiosis in TCM belongs to Lung withered. Dust inhaled into lung deposit in the lung with impairment of lung, and it go against breath. So develop some symptoms such as cough, oppression in chest, short breath and so on ${ }^{[4-6]}$. Lung disease do not improve for a long time that will hurt the spleen and stomach. The deficiency of lung will result in dysfunction of spleen in transportation, and develop some symptoms of spleen-qi deficiency such as shortness of breath and slow statement,floating face and swollen feet and so on. The deficiency of lung-qi cause further the symptoms of kidney deficiency. It follows that the development of pneumoconiosis is the chronic evolution process that deficiency develops in lung, spleen and kidney, and then cause deficiency in five organs; Therefore, dryness-heat, phlegm and blood stasis and weakened body resistance are three key for the pathogenesis of pneumoconiosis ${ }^{[7-8]}$. TCM therapy emphasizes the integration concept, attaches great importance to the overall adjustment in the treatment of diseases, emphasizes the treatment based on syndrome differentiation, the treatment should take the two aspects-cure and treats, Supplement vital energy and eliminate unhealthy influence, according to the feature of the disease, it is a matter of emphasize. And the domestic made great progress in TCM treatment of pneumoconiosis. In the year of 2015-2016, Application of TCM syndrome differentiation in 160 cases of patients with clinical curative effect analysis in our hospital, curative effect is satisfied, report as follows.

\section{Materials and methods}

\subsection{Materials}

Cases source: randomly selected 160 cases of pneumoconiosis patients admitted to Qingdao city center hospital, during the period of 2015.7 to 2016.7. 112 cases of one phase of the pneumoconiosis, 29 cases of two phase of the pneumoconiosis, 19 cases of three phase of the pneumoconiosis. stratified randomly divided into experimental and control group, 102 cases of the experimental group, 58 cases of the control group, approximation ratio 2:1.Cases selected conditions: On the basis of the pneumoconiosis diagnosis standard(GBZ 70-2009) in the diagnosis of pneumoconiosis patients. (1)The patients with hormone and immune inhibitors can't been selected into the groups; (2)The patients who are more than 80 years old or have serious complications can't been selected into the groups; (3)Subjects fill out the questionnaire as requested and sign patients informed consent.

\subsection{Methods}

1.2.1 The control group Given conventional oxygen and nursing therapy, if necessary, select antibiotics to control respiratory infections according to antimicrobial susceptibility test ${ }^{[9]}$.

1.2.2 The experimental group On the basis of the control group, by the syndrome differentiation of TCM, divided into five different syndrome types: 7 cases of dryness-heat injuring lung; 35 cases of phlegm-heat obstructing lung; 9 cases of phlegm and blood stasis; 11 cases of lung and kidney deficiency; 40 cases of lung and spleen deficiency. According to the 
different syndrome types with the corresponding medicinal broth.

\subsubsection{The observed indexes The change of} signs and symptoms that include cough, sputum, chest tightness, chest pain, dyspnea, lung rale and so on before and after treatment; SGRQ each score and total score, SGRQ table is applied to assess the quality of life(QOL) in patients with chronic obstructive pulmonary disease(COPD) in our country, the results show that the table has a good reliability and validity ${ }^{[10]}$; The content of four parameters of fibrosis in serum, before and after treatment; The curative effects judgment of two groups, the judging principle refer to the guidelines of diagnosis and treatment on idiopathic pulmonary fibrosis(mesenchymal $)^{[11]}$.

\subsection{Statistical analysis}

Using SPSS18.0 software for data entry and statistical analysis. All the measurement data were expressed as the Means \pm SD.Analyze count data by chi-square test; Variance test or $\mathrm{T}$ test is used to analyze the measurement data conforms to normal distribution, parameter test is used to analyze the non-normal distribution. Probability values lower than 0.05 were considered statistically significant.

\section{Results}

\subsection{Population distribution analysis}

Two observation group had no difference in age, the length of exposure to dust, gender distribution and pneumoconiosis stage $(\mathrm{P}>0.05)$. All data are shown in Table 1.

Table1 Two groups of population distribution $(\mathrm{n}=160)$

Experimental group $(n=102) \quad$ Control group $(n=58) \quad P$

\begin{tabular}{lccc}
\hline age(years) & $67.94 \pm 8.96$ & $66.40 \pm 9.47$ & 0.951 \\
the length of exposure to & $24.04 \pm 11.63$ & $23.59 \pm 10.16$ & 0.198 \\
dust(years) & 53 & 32 & 0.696 \\
gender distribution & 49 & 26 & \\
male & & & \\
female & 67 & 45 & 0.286 \\
pneumoconiosis stage & 21 & 5
\end{tabular}

\subsection{Laboratory analysis}

At present, According to the four parameters of fibrosis in serum, the changes of pneumoconiosis patients' pulmonary fibrosis could be observed. The content of four parameters of fibrosis in serum had no difference before treatment, the degree of pulmonary fibrosis population distribution had no difference between the two groups. Two groups of patients after treatment, the content of four parameters of fibrosis in serum were 
reduced shown in table 2 , the change of the content of PCIII had difference between the two groups, with statistical significance $(\mathrm{P}<0.05)$. PCIII is closely related to the formation of liver fibrosis, but have no specificity; and when other organ fibrosis, PCIII also rise. After treatment, In the experimental group, the content of PCIII is significantly lower than the control group; Compared with conventional therapy, TCM syndrome differentiation and treatment on pneumoconiosis can effectively inhibit the development of fibrosis.

Table 2 The content of four parameters of fibrosis in serum before and after treatment

\begin{tabular}{lcccc}
\hline & & $\begin{array}{c}\text { Experimental group } \\
\left(\mathrm{ng} \cdot \mathrm{ml}^{-1}\right)\end{array}$ & Control group $\left(\mathrm{ng} \cdot \mathrm{ml}^{-1}\right)$ & P \\
\hline $\begin{array}{l}\text { before } \\
\text { treatment }\end{array}$ & PCIII & $26.195 \pm 10.861$ & $26.282 \pm 11.188$ & 0.961 \\
& IV-C & $28.276 \pm 11.071$ & $27.696 \pm 11.055$ & 0.750 \\
& HA & $75.683 \pm 46.385$ & $75.189 \pm 39.883$ & 0.946 \\
after & LN & $42.886 \pm 25.508$ & $47.417 \pm 16.907$ & 0.228 \\
treatment & PCIII & $22.796 \pm 7.260$ & $25.848 \pm 6.832$ & 0.010 \\
& IV-C & $27.279 \pm 8.521$ & $27.145 \pm 9.181$ & 0.926 \\
& HA & $48.750 \pm 21.392$ & $45.027 \pm 17.059$ & 0.258 \\
& LN & $36.409 \pm 20.419$ & $34.616 \pm 30.194$ & 0.656 \\
\hline
\end{tabular}

PCIII: procollagen type III; IV-C: collagen type IV; HA: hyaluronic acid; LN: laminin

2.3 The analysis of the questionnaire and curative effects judgment

At present, the SGRQ is applicable in patients with Airflow obstructive disease all over the world; The more serious the impact on the life, the higher the weight, the greater the score and the range of 0-100. Two groups of patients had no difference in the respiratory symptoms, limited activity, disease effect and total score before treatment $(\mathrm{P}>0.05)$; Two groups of patients had no difference in the prevalence before treatment. Two groups after treatment, the grade in the respiratory symptoms, limited mobility, disease effect and the total score were reduced; Two groups had difference in the grade of the respiratory symptoms, disease effect and the total score, with statistically significant $(\mathrm{P}<0.05)$. The curative effects judgment by chi-square test, there were differences between the two groups of treatment effectiveness, with statistically significant $(\mathrm{P}<0.05)$; The treatment effectiveness of the experimental group is significantly higher than the control group. The above data are shown in table 3. 
Table 3 The questionnaire of SGRQ and curative effects judgment

\begin{tabular}{|c|c|c|c|c|}
\hline & & $\begin{array}{l}\text { Experimental } \\
\text { group }\end{array}$ & Control group & $\mathrm{p}$ \\
\hline \multirow{4}{*}{$\begin{array}{l}\text { before } \\
\text { treatment }\end{array}$} & respiratory symptoms & $15.784 \pm 3.229$ & $16.241 \pm 3.461$ & 0.403 \\
\hline & limited mobility & $13.353 \pm 2.923$ & $13.793 \pm 3.417$ & 0.391 \\
\hline & disease effect & $18.001 \pm 4.057$ & $18.137 \pm 4.127$ & 0.837 \\
\hline & total score & $47.039 \pm 8.158$ & $48.172 \pm 9.005$ & 0.417 \\
\hline \multirow[t]{4}{*}{ after treatment } & respiratory symptoms & $13.196 \pm 2.541$ & $15.672 \pm 3.495$ & 0.001 \\
\hline & limited mobility & $13.039 \pm 2.583$ & $13.741 \pm 3.312$ & 0.138 \\
\hline & disease effect & $15.961 \pm 3.784$ & $17.259 \pm 3.980$ & 0.042 \\
\hline & total score & $42.196 \pm 6.794$ & $46.672 \pm 8.709$ & 0.001 \\
\hline \multirow[t]{2}{*}{$\begin{array}{l}\text { curative effects } \\
\text { judgment }\end{array}$} & $\begin{array}{l}\text { good reaction or } \\
\text { improved }\end{array}$ & 71 & 20 & 0.001 \\
\hline & poor response or failure & 31 & 38 & \\
\hline
\end{tabular}

\section{Discussions}

Pneumoconiosis is the main occupational disease in our country at present; Not only the quantity of patients is large, and the damage of disease is great, and it lead to a serious loss of labor ability and disability; But also it is the main occupational diseases for national and corporate compensation. Pneumoconiosis is a chronic progressive occupational disease, its pathological changes is diffuse fibrosis of lung tissue, and fibrosis is repairing mechanism after lung injury ${ }^{[12]}$; Theoretically, type I alveolar epithelial cells are not renewable, and the regeneration ability of type II alveolar epithelial cells is also very limited. The principle of its treatment should be: prevent and treat complications actively, delay the progression and relieve patients' pain, prolong the life of patients, improve the quality of life; To achieve these purposes, current treatment is mainly focused on the comprehensive treatment.
At present, the treatment of pneumoconiosis is lack of effective treatment; Western medicine commonly used corticosteroids and immunosuppressant therapy, but the treatment effect is not satisfied, and the side effects is great, the specificity is poor; There is little probability of long-term application. In recent years, many scholars ${ }^{[13-15]}$ used Traditional Chinese medicine for the treatment and intervention to pneumoconiosis ; It is reported that no matter from the disease itself or from relieving symptoms and improving patients' quality of life, TCM have played an important role. Unfortunately, the existing research on mechanism and clinical effectiveness of TCM are mostly related to the small sample survey of individual herbs, individual small combinations, monomer treatment; there is little research on large sample case controlled study of TCM syndrome differentiation. This study uses TCM syndrome differentiation for the treatment of 160 cases of pneumoconiosis; According to the syndrome 
differentiation of TCM, divided into five different syndrome types: Dryness and heat consuming lung, Phlegm-heat obstructing lung, Phlegm and blood stasis, Lung and kidney deficiency, Lung and spleen deficiency; According to the different syndrome types with the corresponding medicinal broth, and combined with the patient's overall physical fitness to add and subtract.

The clinical feature of the syndrome of dryness-heat injuring lung is that body heat, dry cough without phlegm or sputum less and sticky, the blood in phlegm, breathe heavily due to air reversed flow, thoracic fullness and hypochondriac pain, dryness of nasopharynx, thirsty upset, weak breath and tired, red tongue, coated tongue is dry with thin white or thin yellow, pulse count. Its prescription is sangxing decoction with qingzao jiufei decoction. The function of the sangxing decoction is that anti-fever and soothing the lung, moistening lung for arresting cough; The function of the qingzhaojiufei decoction is that anti-dryness and moistening the lung, nourish yin and benefit Qi. Two kinds of prescriptions used at the same time: nourishing Yin and moistening dryness, clearing lung and eliminating phlegm.

The clinical feature of the syndrome of phlegm-heat obstructing lung is that cough, expectoration is yellow viscous and plentiful, oppression in chest, asthma is intense and short, or gurgling with sputum, dysphoria, fever and thirst, or cough up purulent blood and foul sputum, chest pain, bound stool and scanty dark urine, red tongue, coated tongue is yellow and greasy, pulse count. Its prescription is xiebai powder with metal-clearing phlegm-transforming decoction. The function of the xiebai powder is that clearing away the pathogenic heat of lung and relieving cough and asthma; The function of metal-clearing phlegm-transforming decoction is that clearing lung and eliminating phlegm. Two kinds of prescriptions used at the same time: removing heat-phlegm, relieving cough and asthma.
The clinical feature of the syndrome of phlegm and blood stasis is that muscular articulation with stabbing pain, skin is dark purple and swelling, with oppression in chest, tongue is dark purple or with ecchymoses, coated tongue is white and greasy, pulse string. Its prescription is xuefu zhuyu decoction with erchen decoction. The function of the xuefu zhuyu decoction is that promoting blood circulation to remove blood stasis and promoting qi circulation and relieving pain. The function of the erchen docoction is that drying damp and eliminating phlegm and smoothing and reconciling qi in spleen and stomach.Two kinds of prescriptions used at the same time: dissipating phlegm to remove asthma, removing phlegm and blood stasis and activating collateral.

The clinical feature of the syndrome of lung and kidney deficiency is that short breath, expectoration is sticky and frothing, spinning brain and ringing ears, aching lumbus and limp legs, nervous, not resistant to fatigue; or dysphoria with feverish sensation in chest, palms and soles, hectic cheek, dry mouth, red tongue less moss, pulse count; or fear of cold and cold limbs, look pale, coated tongue is light white and fat, deep and thin pulse. Its prescription is lung-supplementing decoction with baihe gujin docoction. The function of the lung-supplementing decoction is that yangyin and soothing lung. The function of the baihe gujin docoction is that nourishing lung and kidney and clearing up the phlegm to stop coughing. Two kinds of prescriptions used at the same time: nourishing the kidney and lung, relieving cough and reducing sputum.

The clinical feature of the syndrome of lung and spleen deficiency is that long coughing, shortness of breath and wheezing, sputum is thin white and plentiful, low sound and slow statement, anorexia, abdominal bloating, thin sloppy stool, look is weary force, look pale, floating face and swollen feet, tongue is light and coated tongue is white, thin and count pulse. Its prescription is lung-supplementing decoction with liujunzi decocotion. The function of the lung-supplementing decoction is that yangyin and soothing lung. The function of the liujunzi decocotion 
is that nourishing qi to invigorate spleen and drying damp and eliminating phlegm. Two kinds of prescriptions used at the same time: tonifying lung and strengthening spleen.

To sum up the above arguments, this study from the perspective of TCM, focus on three key pathogenesis of pneumoconiosis: dryness-heat, phlegm and blood stasis, weakened body resistance; The pneumoconiosis is divided into five different syndrome types, according to the different clinical symptoms and characteristics of syndrome types, adopt corresponding effective prescription. Although the observation time is shorter, results show that TCM syndrome differentiation and treatment has certain inhibitory effect on the progress of pneumoconiosis; Delay the development of patients ' condition, have the effect on easing patients' clinical symptoms, and have no obvious adverse reactions.

\section{References}

1. Wang Jing-fan, Jin Li-hua, Wang Zhan-hong, et al. Traditional Chinese Medicine Discriminate Treatment Pneumoconiosis: Analysis of 188 Cases[J]. China Foreign Medical Treatment, 2008, 27(15): 3,21

2. Goldyn SR, Condos R, Rom WN. The burden of exposure-relateddiffuse lung disease[J]. Semin Respir Crit Care Med, 2010, 29(6): 591

3. Morrison C, Davey G. Assessment of respiratory function in pa-tients with podoconiosis[J]. Trans R Soc Trop Med Hyg, 2009,103(3): 315

4. Jia Xin-hua, Chen Xuan-hai, Zhang Xing-cai, et al. Pulmonary Fibrosis According to"Arthralgia"Theory $[\mathrm{J}]$. Information on Traditional Chinese Medicine, 2013, 30(20): 1-3

5. Wang Li, Ma Zhi, Sheng Li, et al. Development of study on Traditional Chinese Medicine in treatment of silicosis[J]. Chinese Journal of Industrial Medicine, 2005, 18(5): 292-294
6. Song Jian-hua. Ancient and Modern Discourses on Bi-syndrone of Lung[J]. Chinese Journal of Medical History, 1998, 4(4): 212

7. Wang Xue-tao, Chen Yu-zhu, Liu Li-hua. Treatment of pneumoconiosis with TCM syndrome differentiation[J]. China Medicine, 2010, 5(4): 318-320

8. Kao Xi-liang, Zhang Wei. Discussion of the Etiology and Pathogenesis of Pulmonary Interstitial Fibrosis in Terms of Phlegm in Traditional Chinese Medicine[J]. World Journal of Integrated Traditional And Western Medicine, 2012, 07(6): $523-525$

9. Li Guang-jie, Xie Song-jian, Tian hong, et al. Application research on the treatment of coal workers' pneumoconiosis with integration of traditional and western medicine[J]. China Occupational Medicine, 2011, 38(4): 316-317

10. Fang Zong-jun, Cai Ying-yun, Jiang Hao-ming, et al. The SGRQ questionnaire assessment of quality of life in patients with chronic obstructive pulmonary disease[J]. Chinese Journal of Behavioral Medical Science, 1996, 5(1): 16-17

11. The Chinese medical association epidemiology branch of breathing. The guidelines of diagnosis and treatment on idiopathic pulmonary fibrosis(mesenchymal)[J]. Chinese Journal of Tuberculosis And Respiratory Diseases, 2002, 25(7): 387-389

12. Wang Xue-tao, Liu Li-hua. Study on Anti-pulmonary Fibrosis Mechanism of Chinese Herbal Medicine[J]. Occupation And Health, 2010, 26(1): 84-85

13. Ju Hong-mei. Clinical observation on treating 127 cases of CWP in the integrative medicine[J]. Clinical Journal of Chinese Medicine, 2015, 7(7): 112-113

14. Wang Jing-fan, Jin Li-hua, Wang Zhan-hong, et al. Traditional Chinese Medicine Discriminate Treatment Pneumoconiosis: Analysis of 188 Cases[J]. China Foreign Medical Treatment, 2008, 27(15): 3,21

15. Li Guang-jie, Xie Song-jian, Tian hong, et al. Application research on the treatment of coal workers' pneumoconiosis with integration of traditional and western medicine[J]. China Occupational Medicine, 2011, 38(4): 316-317 